\title{
A Discourse about Autism Spectrum Disorders from the Aspect of Gender Relevancies of Biopsychology and Brain Research
}

\author{
Lehel Simon* \\ Levente Szilagyi Entactex Pharma Research Project, Hungary \\ *Corresponding author: Lehel Simon, Hungary
}



\begin{abstract}
Asperger-autism (in its earlier name "autistic psychopaty”) has been scientifically considered as a type of „male-brain disorder" since 1991 (BaronCohen theory). One reason for this is that these patients produce extremely low results, their divergent functions are hindered and the proportion of male-female is approximately 6:1 with this disease. Our article though mainly focuses on the childhood psychotic autists (thinking of Kanner syndrome and Rett-syndrome) with whom the convergent functions are damaged and divergent functions even more looking at them from a personality psychological perspective.

The lack of emotional relations is not replaced by the "savant" characteristics (see Asperger syndrome) but by their immersing into stereotypic instincts. In other words: they compensate their divergent intrinsic emotions with imitated convergences (eg: the monotonus "convergence obsessed" logic of hammering, wringing hands etc.) Today's science cannot declare this to be convergent, especially for the fact that psychotic autist patients prove to be weak at convergence, but the male/female proportion reflects on notable facts (with Kanner syndrome it is 3:1 or 4:1, while Rett syndrome only affects females) as well as the lack of positive divergence (eg: emotional adaptation). An even more blatant fact is the inability to produce real cognitive convergence ant its replacement with instinct convergences (in our hypothesis this is the stereotypic element in autism); and because it is an obsessional instinct driven by the unconscious, it corresponds divergence in the classic view. Can we declare Kanner and Rett syndromes to be basically female brain disorders? We are looking for an answer to this in our paper with a discussion about the functional applicableness of LORETA- EEG, fMRI and FDG-PET-MRI, opening a new approach in autism research.
\end{abstract}

Keywords: Autism; Brain hemispheres; Gender

\section{Discussion}

It is common to address autistic disorder as "pervasive" or "comprehensive" ontogenetic disorders because they effect all areas of adolescent psychological development negatively. But as the expression itself suggests we are not aware of the specific disorders directly. As it will turn out we cannot deal with autism as on complex disorder, we should rather use the term in plural i.e. autisms and autistic disorders.

E.g. the Asperger-syndrome (AS) has been in use as diagnostic criterion since 1944; it was named as autistic psychopathia then. It only got the name Asperger-syndrome referring to the first diagnostician from Lorna Wing in 1981. After all it was only involved in the official system of DSM-IV as a unique syndrome in 1994 and it is still much debated etiologically and from other aspects [1].

It is interesting and its importance will be emphasized later that boy: girl occurrence rate is $6: 1$, and it does not show symptoms in early childhood while other autisms do so. Its real occurrence is frequently met after the age of six. It is considered as child psychopathia by clinical child psychologists even today and no etiologic relation is seen between psychotic and neurodegenerative autisms. The fact that the IQ of children with AS is usually outstandingly high-often exceeds the value 140-further supports this idea. We are going to point out that the classification spread in the '90s was totally wrong by saying that this is a mild form of autisms as opposed to the seriously retarded Kanner-syndrome which was considered as a severe form of autism in this old classification. After 1994 we cannot even talk about such a division $[2,3]$.

It is much debated whether it exclusively hits boys. There are many arguments which say that we tend to pay more attention to boy gender samples, especially when they show extreme intellectual capacity. Though the above mentioned prevalence rate is generally accepted: it is unquestionable as for the contemporary statistical data. Its frequency is $2 / 10000$ (though as we know the prevalence 
of every autistic clinical aspect has dramatically grown in the last 20 years, these are also official data from 1995 further on) [4].

If we take a look at the latest psychological function theory of AS (Baron-Cohen EQ-SQ theory) the male -female rate proves even more to be right, functionally too. This is because besides the extremely low EQ and extremely high IQ and SQ AS strengthens the convergent comprehensive function so it results in a high organizational intelligence and in a lower emotional intelligence adversely. So the convergent male-hemisphere function is extremely dominant and the divergent feminine functions are not on only incapable of communication with the convergent functions but they are behind [5].

If we look at the above described model form the aspect of psychodynamics including emotionality it seems to be easy to establish a psychoactive model as for which there is a psychopathic scission between the systematic and unsystematic cognitive, emotional and personality-structure-functions [6]. This gives a good example for the more and more accepted model which say that children with AS are likely to become adolescents or adults with borderline personality disorder.

We do not yet hold credible statistical data about this, but more and more psychodynamic system states that there is successive relation between childhood psychopathia with AS and adulthood psychopathic pathographies especially borderline syndrome. It would be a mistake though to apply the so called "minified adult model"; AS is a unique pathography of psychopathia. Its drug therapy has not been worked out even in theory, it will also be discussed later here [7].

The zero-LORETA 3D EEG examination method could serve as a brand new evolutionist examination, which is much better in localization than any other common EEG-asymmetria examination [8]. FMRI examination that is even better in localizing this function and the corpus callosum MRI on big population can gain great significance; we would like to integrate it by the FDG-PET-MRI examination

The old and outdated misbelief which says that the childhood autism is a special relapse (shub) of adulthood schizophrenia is absurd and has never even been documented. It is quite obvious that the childhood psychotic autisms (low IQ-often under 75, perinatal neopathia possible) often show a different pathography compared to childhood schizophrenia (relatively high IQ, no perinatal neopathia) .This can be well distinguished Psychopathologically and dynamically from autistic-psychopathia or from borderline syndromes evolving in late childhood which are basically psychopathic with occasional psychotic relapses (shub) as described by modern etiologic models.

In all the DSM classifications traditionally Kanner Syndrome, which we have known since 1943, counts as the childhood psychotic autism. It is such a standard autism that its discoverer's name is not even noted by the DSM-IV but we will see that it is only a type of severe autistic disorder. Mental defectiveness is $100 \%$ in this case, IQ is under 75 and the pervasive disorder can be recognized before the age of three in communication, social behavior and in flexible thinking. Patients often become echolalic, are incapable of communication even on motor level and the stereotypic behavior in psychomotorics. They cannot recognize the personal pronouns and possessive pronouns or invariably repeat them; meaningless, ritual behavior patterns are often. The syndrome's incidence 3-4/10000 and it is increasing, but the most interesting fact is that the girl: boy rate is $3-4: 1$. Unambiguous fallback can be recognized in the above mentioned areas compared to healthy coevals before the age of three while they produce the abovementioned pathologic symptoms. This fact will be of greater importance later in our methodic. Unambiguous neurodegenerative reasons for the Kanner Syndrome are unknown $[9,10]$.

On the other hand the Rett Syindrome which is recently commonly classified as a type of autistic disorder has an unknown etiology but it is unambiguously a neurodegenerative disease, it only appears with girls and its incidence is 1:15000. Growth seems to be normal until the age of one then the speech and motor skills show a fallback and microcephalia appears. This can cause brain-stem ataxia and minor hand tremor in its early state. Most patients suffering the syndrome produce sigh like breathing with intermitting apneic periods which are accompanied by cyanosis. The stereotypical hand fumbling is very common the spontaneous and aimed hand movement disappears. Interestingly this symptom does not evolve before the age of 2 or 3. Strong epileptic form generalized tonal-clonal seizures emerge at most of the patients in the early period. These can be well treated by antiepileptic drugs though dystrophy and defective weight gain can emerge. The autistic behavior is general which proves to be curious because the initial severe neurologic pathography, which decays, is accompanied by a permanent psychiatric pathography.

Today we know that those children who suffer Kanner-or Rett syndromes become mentally retarded people with defective social skills in their adulthood. We also know that development therapies in childhood or adolescence may evoke improvement; thus the lack of such therapies or their application make a big difference in patients lives. Today the progression cannot be valuably changed by drug application (this will be detailed later on) but the patients do not develop schizoid personality structures their autism become standardized.

It is quite curious to decide what to consider the Rett Syndrome on the basis of classification. The Nelson Textbook of Pediatric classifies it as "Neurodegenerative disease of various reason" while DSM-IV classifies it as autism or autistic pathography. Clinical child psychologists have only started to discover this syndrome in their field. If we typically look at the common or at least psychodynamically analogous symptoms, which make it possible at all to consider autisms here, then we can create different dynamic categories.

\section{Emotional autism}

With the Asperger Syindrome this is shown in the psychopathic scission between system aticness and un system aticness i.e. a 
typically convergence dominant emotional function phenomenon. All this is severely diverged with the Kanner Syndrome i.e. it rejects every convergent function i.e. echolalia, stereotypic psycho motor, defective use of personal pronouns. This pathological divergence appears as neuro degeneration with the Rett Syndrome patients; this is interesting also because, we do not know what it was before.

\section{Cognitive autism}

It is obvious here that the cause is convergence predominance with Asperger patients while Kanner and Rett patients functionally incapable of basic convergences like complex speech, aimed communication motivated movement etc.

\section{Interactive deficit autism}

This is obviously A\&B

\section{Excited autism}

It is implied that with Asperger patients the psychopathic emotional range of system aticness - un system aticness scission can be observed which can later manifest in various acting-out behavior. It must not be confused with adulthood psychopathia not even in methodical sense. It is more common here that the psychopathic excitation is covered by the emotional bizarreness of the extreme intellect. With the Kanner Syndrome the excitation can be considered as a negative stereotypic psychotic aggression seizure, though we know very little about its etiology because the psychotic status of retardation as opposed to the numerous life story accounts recorded with as per geroid patients. Excitation with the Rett Syndrome is neurologically initiated which becomes a chronic psychotic autistic standard status; see above. The emotional and intellectual excitation of both the Kanner and the Rett syndromes show a very divergent feature, in certain cases the convergent intellectual functions cannot even be recognized.

\section{Stereotypic autism}

See: C\&D Our researchers' discovery We have said that this is a convergence-divergence balance failure, dominantly "boybrain breakage" with the Asperger Syndrome, and we have proved psycho-dynamically that the Kanner and even more the Rett syndromes are divergent "girl-brain breakage" or at least they show such pathological brain hemisphere dominance. This is why we suggest applying the above mentioned diagnostic imaging and electrophysiological examinations here as well; results are predicted to be analogous but adverse in meaning. If possible it is worth to expand EQ and SQ tests along with the IQ test and to apply complex convergence-divergence tests, though there seems to be not much chance for this except for with AS patients; not counting the standardized, approved and generally applied IQ tests.

From genetic aspect we know that autisms and autistic syndromes often overlap with the so called "X-Fragile syndrome" (Martin-Bell Syndrome) the genetic etiology of which we do not know. This scientific case is even more absurd because we cannot undoubtedly identify it with autistic syndromes. We only know that there is such a parallelism, but this is a blurry fiel both in genetics and child psychiatry, more to that we do not know the exact dynamics of these syndromes in psychology. Neither do we know the genetic origin of premutational X-fragile cases or their etiology. We have worked out a specific examination method in our human research plan which can unambiguously falsify the above mentioned phenomena, problems.

We basically know that X chromosome disorders can cause hypothalamus laesio and since the time of modern diagnostic imaging we also aware that severe hypothalamus laesio and corpus callosum laesio can be identified with many childhood autism patients. We deal with the relation of these in another chapter. We can talk about a comprehensive autism research along these complex analogy systems from genetics to psychology. Thought these are scientific analogies within which we can build up a complex research by the above mentioned etiologic, progressive diversification of disorder differences within pervasiveness; with the unique and independent discussion of each specificity.

I do not mention the contemporary pharmacology and our new opportunities since this will be topic of the rest of this tender material. Up the cooperation of child psychiatry, clinical child psychology and the special education compensating therapy in existence as there is one with adulthood psychosis or psychopathia. The aim of this article and research is to develop such a biologic psychiatric therapy. It is clear that complex developing therapy cannot be neglected later either.

\section{References}

1. Flusberg TH (1999) Neuro developmental Disorders MIT Press. In: Baron-Cohen S, Tager Flusberg H (Eds.), The extreme-male-brain theory of autism. University of Cambridge, USA.

2. Behrman RE, Kliegman RM, Nelson VE, Vaughan VC (2002) Nelson: a gyermekgyógyászat tankönyve nelson textbook of pediatrics. In: Behrman RE, Kliegman RM, Nelson VE, Vaughan VC (Eds.), Harcourt brace \& company, Philadelphia, USA.

3. First MB, Frances A, Pincus HA (1999) Dsm-Iv Textrevision. In: First MB, Frances A, Pincus HA (Eds.), Animula Kiadó, Hungary.

4. Fitzgerald M, Walker SM (2009) Infant pain management: a developmental neurobiological approach. Nat Clin Pract Neurol 5(1): 35-50.

5. Otto F (1957) Kernberg: OFMD Borderline Conditions and Pathological Narcissism. Jason Aronson Inc., New York, USA.

6. Wing L (1981) Sex ratios in early childhood autism and related conditions. Psychiatry Res 5(2): 129-137.

7. Attwood T (1999) The Pattern of Abilities and Development of Girls with Asperger's Syndrome. Asperger's Syndrome foundation, pp. 1-5.

8. http://livewithit.blog.hu/2008/08/30/az_aspi_olyan_mint_a_hagyma

9. http://www.medterms.com/script/main/art.asp?articlekey=5650

10.www.gyogyinfok.hu/magyar/fekvo/hbcs50/torzsek/ BNOTORZS_20060701.xls 